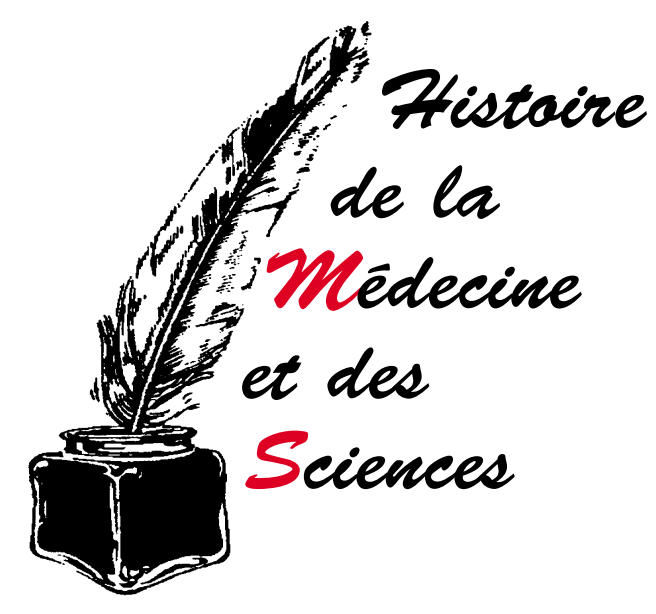

\title{
Naissance
de la biomédecine, le point \\ Naissance
de la biomédecine, le point de vue d'un historien
}

La biomédecine est un aboutissement. C'est la rencontre de deux disciplines : la médecine qui est une application des sciences au maintien de la santé humaine et la biologie qui est, au sens moderne du terme, une science dont la démarche est d'expliquer le fonctionnement de la vie au niveau cellulaire. Cette rencontre ne s'est faite que partiellement dans une logique d'avancée scientifique. Pour que la biomédecine apparaisse, il a fallu surmonter la prévention des médecins envers la prétention des biologistes à expliquer l'ensemble des mécanismes vitaux, mais également la condescendance des biologistes vis-à-vis de ce qu'ils estimaient être l'incompétence des médecins. L'historien constate que cette rencontre s'est opérée à différentes époques selon les pays et assez tardivement en France. C'est pour célébrer l'événement que le Pr Jean Bernard intervenait, en 1968, dans un colloque organisé par l'OMS sur le thème «Politique scientifique et recherche biomédicale»: «Il ne paraît pas indispensable de remplacer le terme honorable et classique de recherche médicale par celui récemment formé de recherche biomédicale qui a l'inconvénient d'être un barbarisme étymologique avec sa tête grecque et sa queue latine, et qui n'a aucun avantage, car on ne peut concevoir une recherche médicale indépendante de la vie.../ Le langage employé néglige la fécondation donnée à la recherche fonda- mentale par les données cliniques, l'impulsion, la nourriture de ces recherches théoriques par des faits observés sur l'homme.» [1]

Au-delà du barbarisme fustigé par Jean Bernard, cette citation suggère une interrogation sur la nature de la médecine, son dualisme entre savoir et savoir-faire et évidemment sur la nature de la recherche médicale, donc les circonstances de l'apparition d'une médecine scientifique. On verra comment celle-ci résulte d'un effort concerté d'abord de mobilisation de chercheurs et de moyens dans des grands instituts de recherche, ensuite d'une réforme de l'enseignement médical - ce que l'on résume parfois sous le vocable général de politique scientifique.

Naissance de la médecine scientifique

Incontestablement, la France du XIX ${ }^{e}$ siècle a joué un rôle clé dans la naissance de la médecine scientifique. La première étape est la naissance de la clinique pour reprendre le terme de Michel Foucault, grâce à l'invention du stéthoscope par René T. Laënnec et au développement de l'anatomopathologie à l'hôpital. La clinique devient une sorte de laboratoire naturel [2].

Ensuite, Claude Bernard, élève de François Magendie et chef de service à l'Hôtel-Dieu puis professeur au Collège de France - et l'un des fondateurs de la Société française de biologie en 1848 - publie sa fameuse Introduction à l'étude de la médecine expérimentale (1865) dans laquelle il propose de fonder la scientificité de la médecine sur la physiologie. Or, si son nom est resté au Panthéon de la science, Claude Bernard a eu peu de postérité immédiate, au moins dans le milieu médical français. La principale critique faite à la physiopathologie est d'avoir considéré les phénomènes de la vie chez l'homme comme assimilables à ceux des autres espèces. A cet égard, le scepticisme des médecins vis-à-vis de l'expérimentation animale est singulièrement éloquent. En réalité, la physiologie a davantage intéressé le Collège de France que la Faculté de médecine.

Louis Pasteur est le troisième acteur dans l'histoire de la médecine scientifique. On le sait, Pasteur était chimiste et non médecin, mais on connaît son apport capital aux sciences de la vie avec la microbiologie et l'installation des laboratoires près de l'hôpital. Or, les critiques qu'il subit ne sont pas moins vives que celles proférées contre Bernard, certains médecins de l'époque fustigeant la microbiologie comme: «une théorie échappée des cornues d'un chimiste.../ une microbiatrie d'importation germanique.../ un véritable choléra intellectuel» [3].

En fait, la microbiologie se développe dans un institut qui porte le nom du fondateur, mais l'Institut Pasteur fondé à Paris en 1888 aura des rela- 
tions parfois complexes avec la Faculté de médecine. Prenons l'exemple de la santé publique dont le développement doit beaucoup aux travaux de Pasteur (hygiène, médecine préventive, etc.). Si la médecine de santé publique ne connaît qu'un développement laborieux en France, il semble que ce n'est pas tant, comme le disent certains, parce que l'Institut aurait failli à sa tâche [4], qu'à cause de l'indifférence des pouvoirs publics ou de l'attitude négative du corps médical. W. H. Schneider et les historiens signalent le rôle primordial de la Fondation Rockefeller se substituant à l'administration française dans le financement de la santé publique et notamment de la lutte antituberculeuse dans l'entre-deuxguerres [5]. De même, à la fin des années 1930, l'hostilité médicale à l'obligation de la vaccination antidiphtérique-antitétanique vaut au découvreur du vaccin - le pasteurien Gaston Ramon lorsqu'il intervient dans le débat - de se voir prier de retourner à ses chères études. Car Ramon n'est pas médecin, mais vétérinaire.

Bien sûr, cela n'empêche pas l'Institut Pasteur de devenir l'un des principaux acteurs dans l'histoire de la nouvelle biologie, ce qu'illustre la carrière d'un pasteurien emblématique du Xx ${ }^{\mathrm{e}}$ siècle, André Lwoff. Au début de sa carrière, Lwoff travaille sur la lyse, un phénomène qui voit la modification d'une bactérie mise en présence d'un virus (bactériophage), ce qui le conduira au phénomène de l'induction du "prophage» et au Prix Nobel de 1965 partagé avec Jacques Monod et François Jacob. La découverte que l'inducteur du prophage peut être un provirus (cancérigène) mènera Lwoff aux problèmes généraux de l'infection virale et à terminer sa carrière comme directeur de l'Institut de recherche sur le cancer (Villejuif) [6]. Mais là aussi, ce type de recherche s'est développé sous l'œil rien moins que critique de certains médecins qui daubent ces biologistes incapables de leur montrer la réalité physique d'un gène. Il est vrai qu'il s'agit de molécules demeu- qu'au développement de l'instrumentation (diffraction par rayons $\mathrm{X}$, microscopie électronique), c'està-dire à la fin des années 1930 lorsque naît une nouvelle discipline, la biologie moléculaire. Ses implications intéresseront la médecine plus $\operatorname{tard}^{1}$.

En résumé, si la biologie est née dans des grands instituts de recherche et si des médecins français ont créé la clinique, en revanche ces derniers semblent avoir boudé les étapes ultérieures de la médecine scientifique que sont la physiopathologie et la microbiologie.

\section{Médecine et biologie, un réductible dualisme}

Cela explique le retard de la médecine française au cours de la première moitié $\mathrm{du} \mathrm{XX}^{\mathrm{e}}$ siècle. Mais, outre les raisons qu'on vient d'évoquer, il en existe d'autres parmi lesquelles une conception dualiste de la médecine qui n'est pas spécifique à notre pays, mais qui s'y affirme avec plus de véhémence qu'ailleurs. Ainsi, dans un article consacré à la modernisation de la recherche, en 1954, un médecin n'hésite pas à affirmer: «...(qu') il faut se garder de confondre science médicale et art médical, application pratique de cette science. Deux réalités aussi distinctes que sont, en matière de cinéma, la science photographique et l'art de la prise de vue.../ La biologie règne désormais en maîtresse absolue, écrit le Dr Gilbert-Dreyfus. Mais faudra-t-il que notre médecine française finisse par perdre son caractère de médecine individuelle d'inspiration humaniste, qu'elle cesse d'être ce colloque singulier entre un malade et son médecin? Qu'on ne s'y trompe point, la toute-puissance de la biologie ne signifie pas pour autant l'agonie ni même la déchéance de la clinique. En pathologie, le psychisme du patient (aura) toujours son mot à dire

\footnotetext{
1 Le terme de Molecular Biology est inventé par le responsable de la division des sciences naturelles de la Fondation Rockefeller, Warren Weaver [7,
} 87 . qu'ignorent inévitablement le vétérinaire et l'expérimentateur...» [9]. A l'évidence, cette opposition entre l'impersonnalité de la biologie et le dialogue du médecin et de son patient a longtemps exprimé la déontologie de la médecine française. C'est dire qu'elle légitime une pratique qui repose davantage sur le savoir-faire du praticien que sur son savoir, tout court. On ajoutera qu'elle sert aussi de paravent à la défense d'intérêts corporatistes. De plus, ce dualisme a pu constituer un frein à la «scientifisation» de la médecine, ce que l'on perçoit à travers les avatars de l'enseignement médical français. D’une part, les facultés de médecine coupées de l'hôpital ne délivraient qu'un enseignement scientifique de qualité médiocre. En 1893, un certificat de sciences physiques, chimiques et naturelles (PCN) est introduit dans le cursus, remplacé par un PCB - le «B» pour biologie - mais seulement en 1934 [10]. D'autre part, la formation hospitalière, purement clinique, offrait une pédagogie dont l'objectif était moins de cultiver la recherche que de décerner des brevets d'aptitude professionnelle. Les concours d'externat-internat étant d'ailleurs, parfois, utilisés à se constituer de fructueuses clientèles privées.

Or, loin d'être irréductible ce dualisme médical n'a pas empêché une véritable révolution intellectuelle de s'opérer dès l'aube du $\mathrm{XX}^{\mathrm{e}}$ siècle dans certains pays. L'idée que la médecine doit bénéficier en priorité des progrès de la biologie, donc que cette dernière peut constituer l'épine dorsale de la formation des médecins a surgi en Europe centrale. L'Allemagne - ses universités et ses instituts - est devenue un pôle d'attraction pour les étudiants en médecine du monde entier, par exemple pour environ quinze mille jeunes Américains qui fréquentent ses écoles de médecine entre 1870 et 1914. Mais c'est en Amérique du Nord, et grâce à l'activité des grandes fondations scientifiques, que se formalise le schéma d'un enseignement médical moderne conçu pour intégrer les tâches de soins, d'enseignement et de 
recherche, selon une disposition qui prévoit de réunir en un même lieu un hôpital, une université et des laboratoires. Aux États-Unis, le mouvement s'amorce dès les années 1890 à la faculté de médecine de l'université Johns-Hopkins (Baltimore) sous l'impulsion de son dynamique doyen, le PrWilliam Welch, un pathologiste de formation germanique. Parmi ses élèves, les "Welch Rabbits», le DrSimon Flexner prend la direction du Rockefeller Institute for Medical Research (RIMR). Cet institut, fondé à New York en 1901 sur le modèle de l'Institut Pasteur, est l'une des premières réalisations philanthropiques du magnat du pétrole John D. Rockefeller Sr. Comme son homologue parisien, le RIMR réunit des laboratoires de recherche, aux côtés d'un hôpital construit en 1910, pour développer la nouvelle biologie. Dans l'entredeux-guerres un médecin-chercheur, Oswald Avery, y étudie l'acide désoxyribonucléique (ADN) dont il mettra en évidence le rôle comme vecteur du message génétique (1944), mais on note qu'il travaillait, au départ, sur les agents infectieux de la pneumonie.

La nouvelle médecine suppose également une réforme de l'enseignement médical. A la veille de la Première Guerre mondiale, le frère de Simon Flexner, Abraham, avait réalisé, à la demande d'une autre grande fondation scientifique (Carnegie), une enquête sur l'enseignement médical en Amérique du Nord et en Europe. Dans son rapport, Abraham Flexner préconise de fondre l'université dans la clinique, c'est-à-dire de sédentariser à l'hôpital des médecins-enseignants-(chercheurs) : "...bedside teaching by full time practitioners» $[11,12]$. Ce programme est adopté par le General Education Board de la Fondation Rockefeller. Créée en 1913, la Fondation décide de fournir les moyens nécessaires aux universités qui accepteront de mettre en œuvre le plein-temps hospitalier. La première à accepter est l'université Johns-Hopkins qui reçoit 1,5 million de dollars. Elle est bientôt suivie des principales facultés de médecine d'Amérique du Nord (université Washington de Saint
Louis, université de Yale, université de Chicago, université MacGill (Montréal) au Canada, etc.), mais avec quelques exceptions notables comme la prestigieuse école de médecine de l'université de Harvard.

En Europe, la Fondation Rockefeller est à l'origine du Medical Research Council britannique (MRC, 1919), un organisme public dont la tâche est d'installer des laboratoires à l'hôpital, parmi lesquels celui du Dr Alexander Fleming (université de Londres) où la pénicilline sera découverte. Au début des années 1930 dans les pays anglo-saxons, la science médicale se trouve posée sur le piédestal d'où elle ne descendra plus, écrit un historien américain; la Fondation Rockefeller a dépensé près de cent millions de dollars pour faire de la médecine une science à part entière [13].

\section{Naissance d'une biomédecine, en France}

En France comme aux États-Unis et grâce au soutien des grands établissements que sont les instituts Pasteur ou Rockefeller - l'institutionnalisation de la recherche médicale résulte de la mise en place d'une politique de la recherche qui inclut une refonte des études médicales [14].

Cette nouvelle politique scientifique doit d'abord résoudre la question de savoir à qui confier l'innovation médicale : aux médecins ou aux biologistes? Le Cnrs est fondé en 1939 à la suite des campagnes menées auprès des pouvoirs publics par le physicien Jean Perrin et le physiologiste André Mayer, tous deux fondateurs de l'Institut de biologie physico-chimique créé grâce au soutien, privé, du baron Edmond de Rothschild (1928). Dès ses débuts, le Cnrs installe une section de "pathologie expérimentale», mais celle-ci se heurte bientôt à l'ostracisme des biologistes. A la Libération, dans une note rédigée à l'intention du Cnrs pour proposer l'installation d'une nouvelle section de «biologie cellulaire», le pasteurien André Lwoff avance: «...(qu') il ne saurait être question, pour des raisons pra- tiques, de considérer l'étude scientifique des agents des maladies infectieuses humaines comme une branche de la médecine, pas plus que de rattacher à la mycologie, les travaux sur la pénicilline ou à l'entomologie, les recherches sur la lutte contre les insectes.../ Le fait que la microbiologie n'est pas enseignée dans les facultés des sciences, mais dans les facultés de médecine par des professeurs qui ne sont pas des spécialistes a conduit la microbiologie française vers une crise très grave...", ajoute-t-il [15].

Parallèlement au Cnrs, et deux ans après sa naissance, le docteur Serge Huard, secrétaire d'État à la Santé et à la Famille du régime de Vichy, ouvre un Institut national d'hygiène (INH, 1941). Mais ce second organisme est d'abord chargé de réaliser des recherches en épidémiologie, indispensables à une politique de santé publique, notamment pour améliorer la connaissance de la propagation des grandes «maladies sociales »: tuberculose, syphilis, cancer. Signalons que la Sécurité sociale, créée par les ordonnances Laroque de 1945, fournira à l'INH près de la moitié de ses ressources budgétaires.

Apparaît alors, en France, une nouvelle génération de médecins, décidée à prendre en main l'avenir de la profession, comme l'avait fait celle des «Welch Rabbits» américains. Sous le patronage du pédiatre Robert Debré, un groupe de jeunes patrons des hôpitaux, marqué par les lois antisémites de Vichy et par sa participation à la Résistance, réagit contre le conservatisme à la fois politique et scientifique* $\mathrm{du}$ milieu médical ambiant. Il s'agit de renouveler l'élite médicale en introduisant la recherche biologique dans une pratique hospitalière qui l'ignorait pratiquement jusque-là. Ces néocliniciens, à commencer par le Pr Debré lui-même, ont connu les laboratoires de l'Institut Pasteur et

\footnotetext{
* Par exemple, l'Ordre national des médecins est créé par une loi d'octobre 1940, aux attendus explicites d'exclusion des médecins juifs de la profession.
} 
ils ont été boursiers Rockefeller. Au début des années 1950, ils participent à la modernisation de la vénérable Assistance publique de Paris en créant une Association ClaudeBernard (ACB), un titre qui symbolise l'entrée de la physiopathologie à l'hôpital. Les patrons des premiers laboratoires sont nos néo-cliniciens : Jean Bernard (leucémie à SaintLouis), René Fauvert (hépatologie à Beaujon), Bernard Halpern (allergologie à Broussais), Jean Hamburger (néphrologie à Necker), Raoul Kourilsky (pneumologie à SaintAntoine), Alexandre Minkowski (périnatalité à Cochin), Jean Scherrer (neurophysiologie à la Salpêtrière), Jean-Claude Dreyfus et Georges Schapira (pathologies moléculaires aux Enfants-Malades) [16, 17].

La suite de l'histoire est mieux connue. En 1956, se tient le colloque de Caen où il est débattu de l'avenir de l'université et de la recherche française. Jean Dausset, un élève de Debré et un futur Nobel pour ses découvertes en immunologie (Human Leucocyte Antigene), y présente un projet de réforme de l'enseignement médical consistant à transformer le concours d'internat en troisième cycle universitaire. La mise en ouvre de cette disposition se heurte d'ailleurs à l'hostilité des biologistes [18]. Puis ce sont les ordonnances de décembre 1958 qui modifient en profondeur l'enseignement médical français. Cette entreprise - parfois appelée réforme Debré du nom de son auteur qui ne cache pas son inspiration «flexnerienne» (l'intégration des fonctions soins-enseignementrecherche) et qui est, notons le, le père du Premier ministre [19] consiste à fusionner la Faculté de médecine et l'Hôpital. D'où la création des Centres hospitaliers universitaires (CHU) chargés de généraliser la pratique du plein-temps hospitalier. Elle aboutira malgré de vives réticences du corps médical qui accuse Robert Debré de vouloir "faire périr l'élite »[20], un reproche qui avait été fait à Abraham Flexner, aux États-Unis, une quarantaine d'années plus tôt. Quant à la recherche, elle est deve- nistration scientifique. La Délégation générale à la recherche scientifique et technique (DGRST) et le Comité des sages (CCRST) constituent à eux deux une sorte de ministère avant l'heure puisqu'ils sont chargés de gérer l'ensemble du budget public de la recherche (l'enveloppe-recherche). Cela permet à la DGRST de lancer, en 1960, douze «Actions concertées» dont cinq sont consacrées à la biologie et à la médecine: neurophysiologie et psycho-pharmacodynamie, nutrition, génétique, cancers et leucémies, biologie moléculaire.

\section{L'Institut national de la santé et de la recherche médicale}

On aboutit ainsi à la création de l'Inserm. Dans le fil de la réforme Debré, et à l'instigation de la DGRST, un décret de juillet 1964 transforme le vieil INH en un nouvel Institut national de la santé et de la recherche médicale. Le cancérologue Georges Mathé, l'un des fondateurs de cet organisme, y installe des comités scientifiques spécialisés dont l'agencement est éloquent, au moins en termes de priorités scientifiques. Tandis que l'INH avait pour mission principale la santé publique, la première commission de l'Inserm s'intitule «Pathologie cellulaire et tissulaire, cancérologie », la seconde "Génétique, immunologie et pathologie moléculaire», et ainsi de suite. La recherche médicale, jusque-là synonyme d'épidémiologie, s'identifie désormais à de la biologie et à de la génétique. Tandis qu'avec 50 millions de francs de l'époque, l'INH ne représentait que $0,2 \%$ du budget de la recherche publique en 1947, aujourd'hui les 2,6 milliards de francs du budget de l'Inserm expriment les $2 \%$ de l'effort public consenti au profit de la recherche médicale. Un domaine où cet établissement n'a d'ailleurs nul monopole.

L'effort consenti par la République au profit de sa recherche a donc contribué à rendre à la médecine française le rang qui fut le sien un siècle et demi plus tôt. Et même si la médecine a entre-temps profondément changé de nature, on ne peut que se ranger derrière le propos de Jean Bernard récusant le dualisme fallacieux de l'adjectif "biomédical». Car, s'il est de nos contemporains pour regretter que la médecine d'aujourd'hui ait perdu en humanisme et en «art du dialogue » ce qu'elle a gagné en efficacité technique, on ne saurait oublier les effets négatifs du dualisme médical évoqué plus haut [21]. L'histoire montre au contraire que lorsque la thérapeutique intègre la physiologie, la microbiologie, et aujourd'hui la bio-génétique, toute la médecine progresse d'autant

\section{RÉFÉRENCES}

1. Bernard J. Colloque «Politique scientifique et recherche médicale», Unesco-Oms 26-29 fév. 1968, Arch. DGRST, AN 5 / 890274 .

2. Foucault M. Naissance de la clinique. Paris: PUF, 1988 .

3. Léonard J. La médecine entre les pouvoirs et le savoir. Paris: Aubier, 1981.

4. Zylberman P. L’hygiène dans la République 1877-1916. Thèse U. Paris VII, 1994.

5. Schneider WH. Quantity and Quality, The Quest for Biological Regenaration in TwentiethCentury France. Cambridge University Press, 1991.

6. Girard M. André Lwoff, un novateur. médecine/sciences $1994 ; 10$ : 1215-8.

7. Kohler RE. Partners in Science, Foundations and Natural Scientists, 1900-1945, University of Chicago Press, 1991.

8. Morange M. Histoire de la biologie moléculaire. Paris: La Découverte, 1994.

9. Gilbert-Dreyfus. Le point de vue du clinicien. La Nef (hors série), 1954.

10. Herzlich C, Bungener M, Paicheler G, et al. Cinquante ans d'exercice de la médecine en France. Carrières et pratiques des médecins français. 1930-1980. Paris : Inserm-Doin, 1993.

11. Flexner A. Medical Education in the United States and Canada, a report to the Carnegie Fondation for the Advancement of Teaching, (introduction by H.S. Pritchett), New York, Carnegie Foundation, 1910.

12. Flexner A. Medical Education in Europe. New York, Carnegie Foundation, 1912.

13. Brown ER. Physicians and foundations: the early Rockefeller medical philanthropies, 19011925. Ph D, University of California, 1975. 


\section{RÉFÉRENCES}

14. Picard JF. Poussée scientifique ou demande de médecins? La recherche médicale en France de l'Institut national d'hygiène à l'Inserm. Sciences Sociales et Santé 1992; X. 12.

15. Note signée A. L. (déc. 1944), arch. Cnrs, AN 80284/216.

Cette mise en garde se justifie par la médiocrité de la médecine française de l'époque. Dans les années 1930, par exemple, le projet de construire une nouvelle faculté de médecine à Paris avait failli achopper sur des querelles mandarinales. La Fondation Rockefeller avait proposé 6 millions de dollars à l'Université de Paris à la condition qu'elle consente un effort équivalent de son côté. Mais l'incapacité des professeurs parisiens à s'entendre, ne serait-ce que sur le site où construire la nouvelle faculté, avait conduit la fondation américaine à reprendre sa subvention, tout en portant ce jugement désabusé : «l'enseignement médical français est tellement déficient qu'un docteur en méde- cine dans ce pays en sait vraisemblablement moins que l'un de nos étudiants en fin de première année. La délivrance des diplomes de médecine par l'Université de Paris aux étrangers et aux ressortissants des colonies revient, en fait, à une simple formalité administrative...". Mémo. Coutelen, 10 avril 1934. Rockefeller Archives Center, R.F., 1.1, Série 500 (France).

16. Gaudillière JP. Entre le laboratoire et l'hôpital: biochimistes et biomédecine dans l'après-guerre. Sciences Sociales et Santé, 1992; $\mathrm{X}: 12$

17. Kahn A. Jean-Claude Dreyfus (19161995). médecine/sciences 1995; 11: 929-32.

18. Debré R. Fauvert R, Dausset J. «Un plan de réforme de l'enseignement médical» et intervention d'A. Lwoff dans les débats du colloque de Caen (1-3 nov. 1956), Les Cahiers de la République (hors série), 1956.

19. Jamous H. Sociologie de la décision. La réforme des études médicales et des structures hospitalières, Paris: Éditions du Cnrs, 1969.
20. Debré R. L'honneur de vivre, témoignage. Paris: Hermann, 1974.

21. Pellegrino ED. The reconciliation of technology and humanism: a flexnerian task 75 years later. In: Vevier C. ed. Flexner 75 years later. A Current commentary on medical education, 1985.

\section{Jean-François Picard}

Ingénieur Cnrs, Institut d'histoire du temps présent, 44, rue de l'Amiral-Mouchez, 75014 Paris, France.

\section{TIRÉS À PART}

J.F. Picard. 